\title{
Histopathological detection of lymph node metastases from colorectal carcinoma
}

\author{
Q van Wyk, K B Hosie, M Balsitis
}

\begin{abstract}
Aim-To evaluate whether the assessment of multiple sections from retrieved nodes yields an increased number of metastases compared with the number that would be detected by the commonly applied method of microscopy of a single section of lymph node only.

Methods-A prospective study of 72 colorectal carcinoma resection specimens. Lymph node sampling was based on the current guidelines for the detection of breast cancer metastases in axillary nodes. Lymph nodes up to approximately $5 \mathrm{~mm}$ in maximum extent were processed in entirety, without prior sectioning, and assessed histologically at three levels; larger lymph nodes were processed in entirety as multiple sections and histologically assessed at one level.
\end{abstract}

Results-From a total of 72 carcinomas, eight were Dukes's A, 26 were Dukes's B, and 38 were Dukes's $C$. The mean and median numbers of nodes identified were 13 and 12 , respectively (range, three to 44 ). Of the Dukes's C cases, four contained lymph node metastases identified by our method that might have gone undetected by the current, generally applied method. In one case, this led to the detection of the only nodal metastasis present and therefore "upstaged" the tumour from Dukes's $B$ to $C$. On average, six extra tissue blocks were processed for each case in applying this method.

Conclusion-The assessment of multiple sections of lymph nodes from colorectal specimens leads to the detection of only a small number of additional nodal metastases. The method involves increased workload for pathologists and laboratory staff.

(F Clin Pathol 2000;53:685-687)

Department of

Histopathology,

Northern General

Hospital, Herries

Road, Sheffield

S5 7AU, UK

$\mathrm{Q}$ van Wyk

M Balsitis

Department of Surgery, Northern

General Hospital

K B Hosie

Correspondence to: Dr van Wyk

email: q.vanwyk@virgin.net

Accepted for publication 19 April 2000 specimen. Although guidelines for the pathological assessment of such specimens have been published recently, these do not include recommendations for lymph node assessment, with regard to the number of sections of lymph nodes processed or the number of levels examined from each tissue block. ${ }^{2}{ }^{3}$

The widely accepted practice for pathological assessment of lymph nodes retrieved from colorectal cancer resection specimens is either to select one section from a node and process that section only or to submit the entire node, if it is small enough, without prior sectioning. One level for microscopy is then taken from each tissue block. Although immunohistochemical and molecular methods increase the detection of micrometastases, ${ }^{4}$ such methods have not been adopted as routine practice.

It has been shown previously that the greater the number of lymph nodes sampled, the greater the likelihood is of detecting metastases. ${ }^{16}$ It seems likely that an increased number of metastases would be detected by the assessment of additional tissue and this has been confirmed using a mathematical model. ${ }^{7}$ However, no previous specimen based studies have investigated the effect of processing entire lymph nodes in the setting of colorectal carcinoma.

Specific guidelines exist for the pathological assessment of lymph nodes retrieved from axillary tissue in the setting of primary breast carcinoma. ${ }^{8}$ The aim of our study was to determine whether a similar approach to lymph nodes from colorectal resection specimens would yield an increased number of metastases compared with the current widely applied method. The National Health Service breast screening programme suggests the following method of handling axillary lymph nodes retrieved from breast cancer resection specimens. ${ }^{8}$ Lymph nodes larger than $5 \mathrm{~mm}$ should be cut into three slices and one node should be processed for each cassette, whereas nodes smaller than $5 \mathrm{~mm}$ should be embedded in entirety, and ideally examined at two levels. Axillary lymph nodes tend to be larger than colorectal lymph nodes, frequently allowing multiple sections to be obtained; however, because this method is applied routinely to axillary nodes in our practice, a modification of this method of node handling was applied to colorectal specimens.

We have not attempted to indicate the clinical relevance of the additional metastases detected, but have aimed to determine the extent of metastatic deposits that might be "missed" with current practice. 


\section{Methods}

A prospective study was performed on 72 colorectal resection specimens, removed for carcinoma, taken over a 16 month period (June 1998 to September 1999) in one teaching hospital. The specimens were dissected by two pathologists, QvW and MB. Specimen dissection and tissue sampling of the primary tumour was in accordance with the current guidelines for the histopathological assessment of colorectal carcinoma. ${ }^{23}$ The sampling of retrieved lymph nodes was based on the current guidelines for the detection of breast cancer metastases in axillary lymph nodes as follows. ${ }^{8}$ Lymph nodes were detected by palpation and slicing of associated adipose tissue under a good light. The apical (highest) node was labelled separately when identified. Macroscopically identifiable lymph nodes up to approximately $5 \mathrm{~mm}$ in maximum extent ("small" nodes) were, without further sectioning, submitted in entirety and examined at three levels, at least $100 \mu \mathrm{m}$ apart. Multiple small nodes were processed in one cassette. Nodes larger than approximately $5 \mathrm{~mm}$ in maximum extent ("large" nodes) were divided into at least two sections, up to the maximum number that could be processed in one cassette and examined microscopically at one level. Only one representative section was taken from lymph nodes that were macroscopically replaced by tumour. Particular attention was paid to matching adjacent slices of any nodes transected while slicing the bowel wall for the assessment of the primary tumour. After standard processing, sections for microscopy were cut at $4 \mu \mathrm{m}$ thickness and examined for metastases using a standard haematoxylin and eosin stain only. Further small nodes detected only on microscopy of bowel wall blocks were subsequently examined at two further levels.

The number of lymph nodes containing metastatic tumour was recorded. For involved large nodes, metastatic tumour in any node section other than the larger/largest in a given cassette was regarded as a metastasis that might have gone undetected if only one section of the node had been processed ("additional" metastasis). For small nodes examined at three levels, any metastasis in levels two or three, but not in level one was regarded as a metastasis that might have gone undetected if only one level had been examined ("additional" metastasis). The numbers of involved and uninvolved, small and large nodes were assessed and staging by modified Dukes's stage and TNM classification was applied. ${ }^{9} 10$ The assessment of additional metastases allowed us to determine whether the detection of these metastases led to upstaging of the tumour. To determine the extra workload generated by this method, the extra number of tissue blocks and slides generated for each case was estimated by counting the number of blocks and slides produced and subtracting the estimated number that would have been produced if single sections from multiple nodes had been processed together in one cassette.

\section{Results}

In total, 72 colorectal carcinoma resection specimens were assessed, including eight Dukes's A, 26 Dukes's B, 29 Dukes's C1, and nine Dukes's C2. The mean and median numbers of lymph nodes identified for each case were 13 and 12, respectively (range, three to 44). The mean numbers of lymph nodes from Dukes's A, B, and C cases were seven, 13, and 15, respectively (medians, 11, 11, and 12, respectively). In 52 cases, 10 or more lymph nodes were identified. The single case from which only three lymph nodes were retrieved was Dukes's stage C. For the year before the start of our study the frequency of Dukes's A, $\mathrm{B}$, and $\mathrm{C}$ in resection specimens recorded at our hospital was $12 \%, 47 \%$, and $40 \%$, respectively, and the mean number of nodes retrieved was six, 11, and 10, respectively. The mean number of nodes for all cases was 10 (range, 0-28).

In five lymph nodes from four patients, metastatic deposits were identified that might have gone undetected by the widely accepted method of assessment. In four large lymph nodes from three patients, metastases were identified in only one of the multiple sections of a single node and did not involve the largest slice from the node. In one further case an additional nodal metastasis was found on examining levels of a small node; a metastatic deposit was present in only the second and third levels of the lymph node. However, in only one of the four patients did the detection of an additional metastasis lead to upstaging of the tumour from Dukes's B to C and from N0 to $\mathrm{N} 1$ with regard to TNM classification. This case showed a lymph node metastasis in the smallest of three slices of a large node and was the only metastatic deposit identified in a total of 44 nodes. The lymph node measured at least $7 \mathrm{~mm}$ in maximum extent and the metastasis was present in the smallest of the three slices taken through that node. In the plane examined, this metastasis measured $2.7 \mathrm{~mm}$ in maximum extent. The three other cases described above showed widespread metastases in further nodes; therefore, the additional metastases in those cases did not alter the tumour stage.

With regard to the additional workload generated by this method, an estimated 414 extra tissue blocks were processed (mean, six/case). The approximate number of extra slides was greater (535) because blocks of small nodes had three levels cut, requiring further slides (table 1).

Table 1 Summary of the extra workload involved and additional metastases detected in the study of 72 cases of colorectal carcinoma

Approximate number of additional tissue blocks processed 414 Approximate number of additional slides prepared $\quad 535$ Number of additional metastases detected

Number of cases "upstaged" because of additional metastases 


\section{Discussion}

In our study, five lymph node metastases identified from four colorectal carcinoma resection specimens might have gone undetected if the current widely applied method of lymph node sampling had been used. Although the number of additional metastases detected was small, in one case it led to upstaging from Dukes's B to C. It is not possible to confirm with certainty whether or not the additional metastases detected would have gone undetected if only one section of lymph node was processed, because this would depend on the plane of sectioning by the pathologist. When choosing which piece of a sectioned lymph node to process for microscopic examination, there is obviously an element of chance involved as to whether a metastatic deposit replacing less than half of the lymph node volume will be detected. It has been proved mathematically that the chance of identifying a randomly distributed $1 \mathrm{~mm}$ lesion in a $5 \mathrm{~mm}$ node that is bisected is as low as $37 \%$.

It has been shown that, with regard to colorectal cancer resection specimens, the greater the number of lymph nodes examined, the greater the chances are of detecting metastases in these nodes. ${ }^{1}$ Although it was not the aim of our study to investigate this factor, it is interesting to note that, of the cases studied, 53\% were Dukes's stage C and the mean number of lymph nodes retrieved for each case for this stage was 15, whereas in the year before our study the figures were $40 \%$ and 10 , respectively. Methods have been applied previously to increase the yield of lymph nodes. Scott et al used fat clearance (by means of xylene and alcohol) to increase the number of lymph nodes retrieved from colorectal carcinoma resection specimens. ${ }^{11}$ The five year follow up of patients to whom this method was applied found it to be of clinical relevance. ${ }^{12}$ Fat clearance is relatively inexpensive but is regarded as impractical and unsafe. The health and safety aspect of this technique is the major reason that it has not gained wide acceptance. Both xylene and alcohol are highly inflammable agents and certain occupational exposure limits are laid down for these and other chemicals used in laboratories. ${ }^{13}$ Methods have also been described that increase the detection of occult metastases in lymph nodes, including immunohistochemical staining for cytokeratins and molecular biological techniques. ${ }^{4}{ }^{14}{ }^{15}$ Despite the increased detection of metastases by these methods, they have not been widely accepted as routine practice.

Our study is based on the fact that careful macroscopic specimen assessment still appears to be the most widely accepted means of optimum lymph node retrieval in the UK.

Our results suggest that the number of important (with regard to tumour stage) additional metastatic deposits detected by our method, compared with the usual method of lymph node sampling, appear to be small.
If our method was applied routinely, the time and therefore cost of colorectal carcinoma reporting would be increased for both laboratory and medical staff, although the accuracy of tumour staging might be improved. Given current financial and manpower restraints, it is unlikely that such a method would be widely regarded as appropriate for routine application in view of the low yield of additional metastases. However, pathologists should be aware of the possibility of "missed" metastases and should, at least, select sections from lymph nodes in such a way as to allow the largest possible cut surface to be assessed-for example, by sectioning ellipsoid shaped nodes along their long axes. Lymph node detection is, after all, a time consuming part of specimen assessment and, once identified, each node should be handled in the optimum way for accurate staging. We cannot assess the clinical relevance of the additional metastases detected in our study; however, we have given an indication of the possible extent of metastases missed by processing partial lymph nodes only. Pathologists involved in compiling guidelines for reporting of cancer resection specimens should address the handling of lymph nodes with a view to achieving a uniform approach.

The preliminary results of this study were presented to the Association of Coloproctology of Great Britain and Ireland, June 1999 (Colorectal Disease 1999; 1(suppl 1):25).

1 Hernanz F, Revuelta S, Madrazo C, et al. Colorectal adenocarcinoma: quality of the assessment of lymph node metastases. Dis Colon Rectum 1994;37:373-7.

2 The Royal College of Pathologists. Minimum dataset for colorectal cancer histopathology reports. London: The Royal College of Pathologists, July 1998.

3 UK Coordinating Committee on Cancer Research. Clinicopathological staging booklet. 2nd ed. London: UKCCA, April 1997.

4 Sasaki M, Watanabe H, Jass JR, et al. Immunoperoxidase staining for cytokeratins 8 and 18 is very sensitive for detection of occult node metastasis of colorectal cancer: a comparison with genetic analysis of K-ras. Histopathology comparison with

5 Liefers GJ, Tollenaar RAEM, Cleton-Jansen AM. Molecular detection of minimal residual disease in colorectal and breast cancer. Histopathology 1999;34:385-90.

6 Goldstein NS, Sanford W, Coffey M, et al. Lymph node recovery from colorectal resection specimens removed for adenocarcinoma. Am f Clin Pathol 1996;106:209-16.

7 Wilkinson EJ, Hause L. Probability in lymph node sectioning. Cancer 1974;33:1269-74.

8 Pathology reporting in breast cancer screening. NHS BSP Publication No 3, 2nd ed. Sheffield: NHSBSP Publications, April 1995.

9 Gabriel WB, Dukes CE, Busey HJR. Lymphatic spread in cancer of the rectum. Br F Surg 1935;23:395-413.

10 Sobin LH, Wittekind Ch, eds. TNM classification of malignant tumours, 5th ed. New York: Wiley-Liss, 1997: $66-9$

11 Scott KWM, Grace RH, Gibbons P. Detection of lymph node metastases in colorectal carcinoma before and after fat clearance. Br f Surg 1989;76:1165-7.

12 Scott KWM, Grace RH, Gibbons P. Five-year follow-up study of the fat clearance technique in colorectal carcinoma. Dis Colon Rectum 1994;37:126-8.

13 Health and Safety Executive. EH40/99. Occupational exposure limits 1999. Suffolk: HSE Books, 1999.

14 Hayashi N, Arakawa H, Nagase H, et al. Genetic diagnosis identifies occult lymph node metastasis undetectable by the histopathological method. Cancer Res 1994;54:3853-6.

15 Hayashi N, Ito L, Yanagisawa A, et al. Genetic diagnosis of lymph node metastasis in colorectal cancer. Lancet 1995;345:1257-9. 